\title{
Estado poblacional del saraguato negro (Alouatta pigra) en la Reserva de la Biosfera Pantanos de Centla
}

\section{Population state of black saraguato monkeys (Alouatta pigra) in the Reserva de la Biosfera Pantanos de Centla}

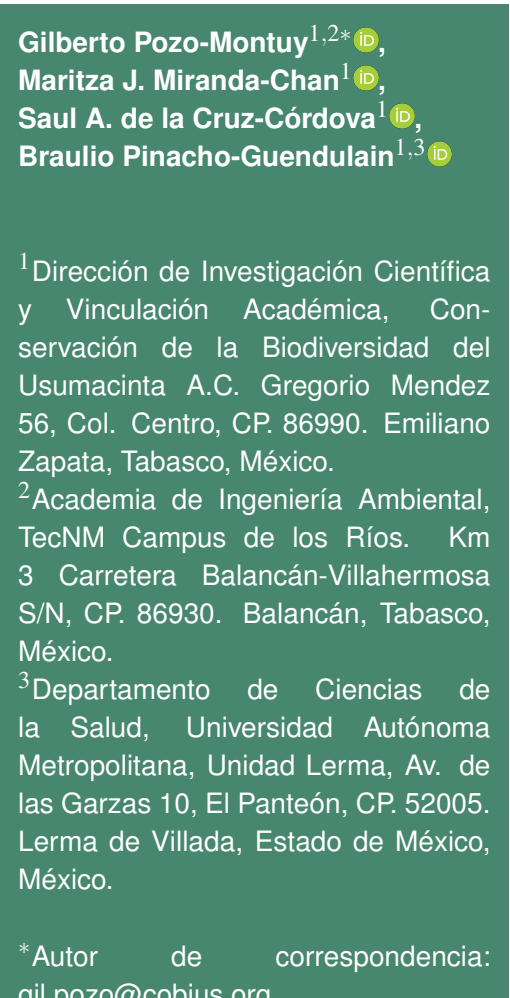

gil.pozo@cobius.org

Artículo científico

Recibido: 05 de julio 2020

Aceptado: 07 de abril 2021

Como citar: Pozo-Montuy G,

Miranda-Chan MJ, de la Cruz-

Córdova SA, Pinacho-Guendulain

B (2021) Estado poblacional del

saraguato negro (Alouatta pigra) en

la Reserva de la Biosfera Pantanos

de Centla. Ecosistemas y Recursos

Agropecuarios Núm. Esp. I: e2672.

DOI: 10.19136/era.a8nl.2672
RESUMEN. La conservación de especies silvestres requiere implementar acciones basadas en el tamaño, composición y distribución de sus poblaciones. Esta información, sin embargo, está incompleta o desactualizada para muchas áreas de gran importancia biológica. El objetivo del presente trabajo fue contabilizar los monos saraguatos (Alouatta pigra) presentes en la Reserva de la Biosfera Pantanos de Centla (RBPC) para determinar su estado poblacional. La RBPC se dividió en tres zonas que fueron censadas en 2015, 2016 y 2018 por seis grupos de monitoreo comunitario. Un total de 1929 saraguatos fueron contabilizados, de los que 57 fueron individuos solitarios y el resto se agrupó en 362 tropas con un tamaño promedio, y desviación estándar, de $4.6 \pm 2.6$ individuos. Más del $60 \%$ de las tropas estuvieron formadas de un macho adulto acompañado de una o más hembras adultas y de individuos inmaduros. La proporción sexual entre machos y hembras adultas fue de 1:1.2 y la proporción de individuos inmaduros por hembra adulta fue de 1:1. Los resultados indican que el tamaño y composición de las tropas presentaron valores representativos para la especie, mientras que los valores de los parámetros poblacionales estuvieron dentro de los intervalos reportados para poblaciones en selvas conservadas y fragmentadas. Este es el primer censo completo de primates realizado en un área tan extensa y de difícil acceso en el que se enfatiza la participación de pobladores locales como una estrategia que ofrece ventajas en los censos de primates.

Palabras clave: Áreas Naturales Protegidas, conteo de primates, fragmentación del hábitat, proporción sexual, tamaño poblacional.

ABSTRACT. Wildlife conservation requires performing actions based on the size, composition, and distribution of their populations. This information, however, is incomplete or outdated for many areas of great biological importance. The goal of this study was counting the Black saraguatos (Alouatta pigra) in the Biosphere Reserve of Pantanos de Centla (BRPC) to determine their population status. The Reserve was divided into three zones that were censused in 2015, 2016, and 2018 by six community monitoring groups. A total of 1929 saraguatos were counted, of which 57 were solitary individuals and the rest of them were grouped into 362 troops with an average size, and standard deviation, of $4.6 \pm 2.6$ individuals. More than $60 \%$ of troops were formed by an adult male, one or more females, and immature individuals. The sex ratio between adult males and females was 1:1.2 and the ratio of immature individuals to adult females was $1: 1$. These results suggest that troop size and composition were representative of the species, while population parameters were within the ranges reported for populations in conserved and fragmented forests. This is the first complete census carried out in a large and difficult to access area, which emphasize the local people involvement as a strategy that offers advantages during the primate population censuses.

Key words: Natural protected areas, primate counts, habitat fragmentation, sex ratio, population size. 


\section{INTRODUCCIÓN}

La mayor preocupación de la biología de la conservación es la protección y continuidad de las comunidades y ecosistemas, así como de los procesos ecológicos y evolutivos que ocurren en ellos (Soulé 1985). Ante la gravedad de los problemas ambientales, la titánica labor de conservar requiere de la participación de especialistas de diversas áreas de la ciencia, de la inclusión de las comunidades locales, y contar con suficiente apoyo económico para emprender acciones de conservación eficaces que se apliquen a corto, mediano y largo plazo; la deforestación, por ejemplo, ha modificado la estructura de las selvas lo que afecta la diversidad y el estado de conservación de las poblaciones de primates silvestres en el mundo (Gouveia et al. 2014, Estrada et al. 2017). Para frenar el impacto de la deforestación se han decretado áreas protegidas en ecosistemas vulnerables con monitoreos longitudinales sobre especies claves o prioritarias (Mouquet et al. 2013, Busch y Ferretti-Gallon 2017), como lo son muchas especies de primates silvestres por su función ecológica como dispersores de semillas (Lambert 2010). En este contexto, la planeación sistemática ha sido también importante para la conservación cuyo objetivo es conocer qué, dónde y cómo conservar, utilizando protocolos para luego establecer acciones de conservación afines al contexto socioeconómico y a la naturaleza del área o de la especie de interés (Margules y Pressey 2000, Rondinini et al. 2006).

México ha desarrollado un sistema de Áreas Naturales Protegidas (ANPs) destinado a la protección y conservación de ecosistemas y especies prioritarias distribuidas en un área de más de 91.5 millones de hectáreas, las cuales representan el 22\% del territorio nacional (Ocampo et al. 2014, CONANP 2018). En cuanto a la conservación de primates silvestres, México ha definido un Plan de Acción para la Conservación de las tres especies que se distribuyen en las selvas húmedas del país: mono araña (Ateles geoffroyi), mono saraguato de manto (Alouatta palliata), y mono saraguato negro (A. pigra) (Oropeza-Hernández y Rendón-Hernández 2012).
En ese sentido, y en un esfuerzo por determinar los principales sitios hacia dónde dirigir las acciones de conservación, varias de las ANPs del sur-sureste de México se sobreponen con los Sitios Prioritarios para la Conservación de los Primates Mexicanos (SPPs), los cuales se identificaron mediante criterios derivados de variables biológicas (presencia/ausencia), factores de presión y de configuración espacial del paisaje (Tobón et al. 2012). Sin embargo, y ante la escasa información, estos criterios omiten la distribución actual y la variación en la abundancia de los monos araña y monos aulladores que, por un lado, permitirían evaluar el estado de conservación de sus poblaciones y, por otro lado, ayudarían en los procesos de priorización y de toma de decisiones.

Ante la falta de información básica para la planeación sistemática de la conservación, es importante emprender esfuerzos en conjunto con la población local reconociendo sus capacidades para el monitoreo biológico. Este modelo inclusivo se ha aplicado en el monitoreo de aves (Ortega-Álvarez et al. 2017) y primates (Calixto- Pérez et al. 2018). En el presente trabajo se presentan los resultados del censo poblacional de monos saraguatos negros (Alouatta pigra) realizado con el apoyo de grupos de monitoreo comunitario a lo largo de tres años en la Reserva de la Biosfera Pantanos de Centla con una extensión de 302706 hectáreas, en Tabasco. Se espera que la población de saraguatos negros en la RBPC sea mayor en los remanentes de selva mediana y selva baja espinosa de tinto que en otras asociaciones arbóreas que ocupan más área o son dominantes en la reserva como es el caso del manglar. Por lo anterior el objetivo del presente trabajo fue realizar un censo con participación comunitaria de monos saraguatos (Alouatta pigra) en la Reserva de la Biosfera Pantanos de Centla (RBPC) para determinar el estado poblacional.

\section{MATERIALES Y MÉTODOS}

\section{Área de estudio}

Pantanos de Centla fue decretado como Reserva de la Biosfera en 1992 y en ella convergen las cuencas bajas de los ríos Grijalva y Usumacinta 
al noreste del estado de Tabasco sobre la planicie costera en el sureste del Golfo de México, en los municipios de Centla, Jonuta y Macuspana (Figura 1). La RBPC ocupa un área de 302706 hectáreas de extensión territorial de la cual un $56 \%$ pertenece a vegetación acuática y subacuática (Guerra-Martinez y Ochoa Gaona 2006). Dentro de su superficie se establecieron en su plan de manejo tres zonas: zona núcleo I con un área de 57738 ha, zona núcleo II con 75857 ha, y zona de amortiguamiento con 170112 ha (Carabias et al. 2000). En general, la flora del área está dominada por comunidades hidrófitas y fragmentos de selva mediana subperennifolia de Bucida buceras, selva baja subperennifolia de Haematoxylum campechianum, selva secundaria, vegetación riparia, y palmares de Sabal mexicana y Acoelorraphe wrightii (Guadarrama-Olivera y OrtizGil 2000, Romero et al. 2000) y en cuanto a su diversidad florística en la RBPC se han registrado 731 especies nativas, 64 introducidas y 18 cultivadas de plantas vasculares (López-Jiménez et al. 2020). Por otro lado, la riqueza faunística está integrada aproximadamente por 633 especies (106 peces dulceacuícolas y marinas, 27 anfibios, 68 reptiles, 328 aves residentes y migratorias y 104 mamíferos), de las que el $25 \%$ se encuentran amenazadas por la pérdida de hábitat y el cambio del uso del suelo (Macossay-Cortez et al. 2011, Barba Macias et al. 2015, Soria-Barreto et al. 2018).

\section{Especie de estudio}

Los saraguatos negros son primates de gran masa corporal del Nuevo Mundo que están provistos de una cola prensil y presentan un desarrollo considerable del hueso hioideo que, junto con el ensanchamiento de la laringe, funciona como una caja de resonancia y amplificador que les permite emitir fuertes aullidos que se escuchan a varios kilómetros de distancia, lo que facilita la comunicación entre tropas (Kitchen 2004, Dolder y Dolder 2009, van Belle y Estrada 2019). El género Alouatta se extiende desde el sur y este de México, pasando por Centroamérica, hasta las selvas secas y deciduas del norte de Argentina (Di Fiore y Campbell 2007). En particular, Aluotta pigra con su característico color ne- gro es endémico de Mesoamérica y cerca del $80 \%$ de su distribución geográfica se encuentra en México, en los remanentes de selva de los estados de Tabasco, Chiapas, y en la Península de Yucatán (Horwich y Johnson 1986, Watts y Rico-Gray 1987, Cortés- Ortíz et al. 2003).

Los monos saraguatos, en general, son muy flexibles conductualmente ante las perturbaciones en el paisaje (Sorensen y Fedigan 2000, BonillaSánchez et al. 2012). Dicha flexibilidad les permite sobrevivir en relictos de vegetación de hasta 0.1 hectáreas en zonas severamente fragmentadas (PozoMontuy y Serio-Silva 2007) y utilizar elementos de la matriz del paisaje para moverse y alimentarse (PozoMontuy et al. 2011). En cuanto a sus requerimientos de energía, los saraguatos utilizan 1165 especies de plantas (Dias y Rangel-Negrín 2015), de las que suelen consumir grandes cantidades de hoja pero, en ciertos meses del año, también pueden consumir un alto porcentaje de frutos silvestres (Dias y RangelNegrín 2015, Pavelka y Knopff 2004).

\section{Conformación de grupos comunitarios de moni- toreo}

Los grupos comunitarios de monitoreo (GCM) se formaron a través de la participación de 27 pobladores locales de diferentes ejidos que conocen su territorio. El monitoreo se realizó entre los meses de julio a noviembre del 2015, 2016 y 2018 luego de contar con la anuencia de las asambleas ejidales: en 2015 se contó con el apoyo del ejido Marcos Díaz y ejido Tijeras; en 2016 participaron Bitzales Séptima Sección, Tembladeras y Rivera Baja; y en 2018 colaboraron El Palmar y Tembladeras. Los GCM recibieron una capacitación teórico-práctica antes de comenzar el conteo de monos. En la capacitación se les explicó la importancia de conservar la especie de estudio, el panorama actual de su conservación, y se les enseñó aspectos importantes de su biología, ecología y comportamiento que pudieran resultar relevantes al tomar decisiones durante el monitoreo. También se les enseñó el uso correcto del equipo de monitoreo (binoculares Eagle Optics 10x42, geoposicionador global Garmin eTrex 10, cámara fotográfica Nikon Coolpix P600, clinómetro), la manera de iden- 


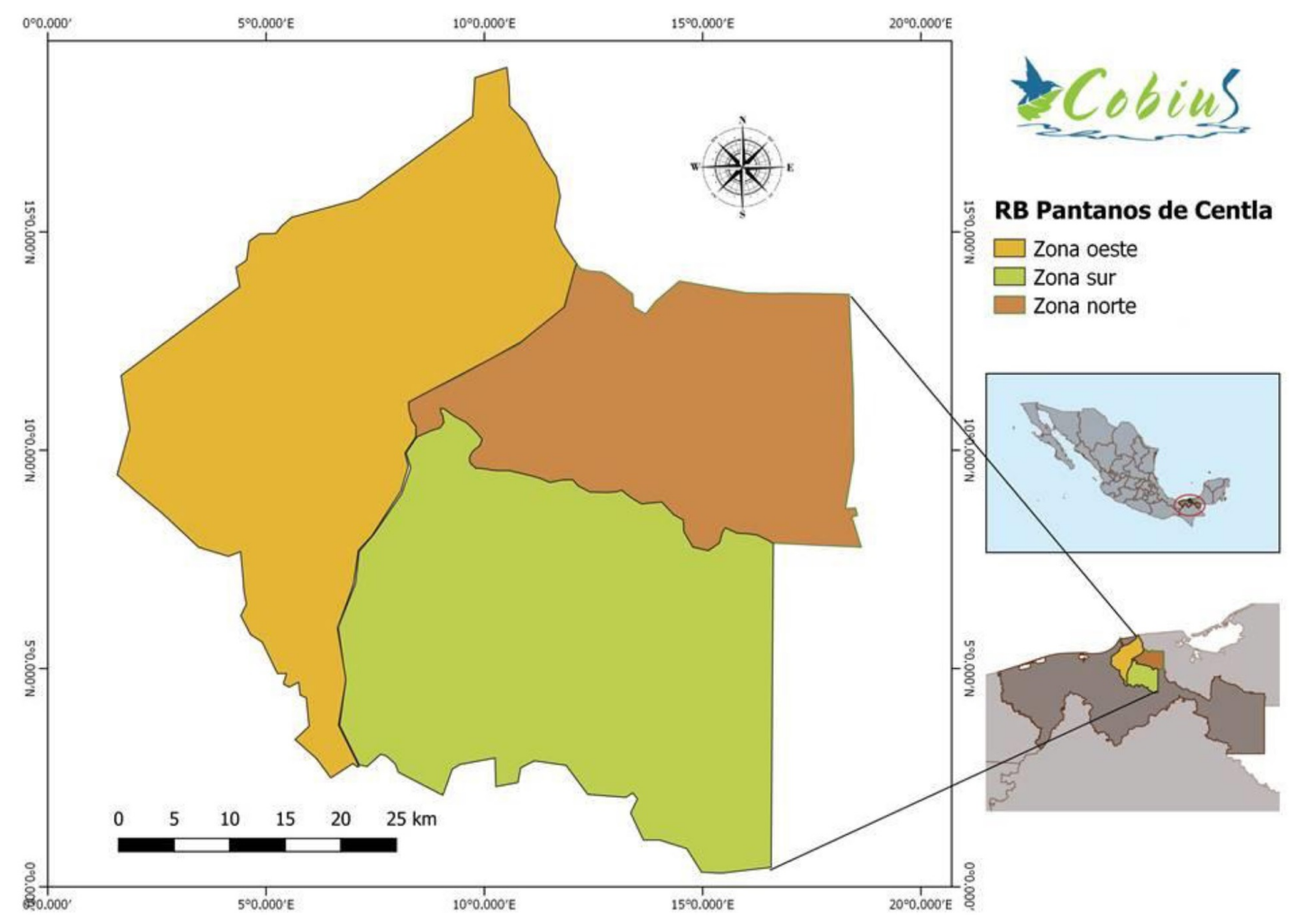

Figura 1. Localización del área de estudio. Los diferentes colores dentro del polígono representan las zonas en las que la Reserva fue dividida para ser censada en años diferentes. Zona norte en 2015, zona sur en 2016 y zona oeste en 2018.

tificar las categorías de sexo y edad de los individuos acorde a los criterios de Pozo-Montuy et al. (2008), y cómo registrar la información en las bitácoras de campo.

\section{Monitoreo poblacional}

Para el conteo total de monos saraguatos negros y con la finalidad de evitar reconteos interanuales la RBPC se dividió en tres zonas (Figura 1) de acuerdo con la presencia de barreras físicas como el cauce del río Usumacinta, popales y tulares, canales, lagunas y arroyos: en 2015 se censó la zona noreste con un esfuerzo de muestreo de $416.87 \mathrm{~km}$ recorridos en 60 días; en 2016 se recorrió la zona sureste con $244.52 \mathrm{~km}$ acumulados en 90 días; y por último, en 2018 la zona oeste con $300.23 \mathrm{~km}$ recorridos en 60 días. Para el conteo total en la RBPC se requirió de un esfuerzo de muestreo de $961.62 \mathrm{~km}$ acumulados en 210 días a lo largo de tres años de trabajo entre los meses de julio a noviembre.
En compañía de uno o más técnicos de campo, los GCM recorrieron los fragmentos de vegetación arbórea de 11 ejidos y localidades ubicadas dentro de la RBPC. El conteo de monos se realizó a través de censos completos. El método consistió en contar todos los individuos presentes en un área determinada, por lo que es apropiado para especies con poca movilidad, que son fáciles de detectar, y que habitan paisajes fragmentados (NRC 1981, Sutherland 2006); condiciones que se cumplen con el mono saraguato negro en la RBPC. Los recorridos para los conteos se realizaron de 07:00 a 18:00 horas a velocidad promedio de $1.25 \mathrm{~km} \mathrm{hr}^{-1}$, pero la velocidad de los recorridos varió en función a las condiciones de la zona. En cada observación de saraguatos, los GCM registraron: número y clave de la tropa, fecha y hora del avistamiento, condiciones del tiempo atmosférico, nombre del ejido, número de individuos presentes, composición de la tropa por sexo y edad, altura y actividad del grupo o de la mayoría del grupo al 
momento del avistamiento (alimentación, descanso, movimiento), ítem consumido (hoja, flor, fruto y tallo), altura del árbol o árboles en los que se encontraban los individuos, posición geográfica, y cualquier otra observación relevante. Cada registro duró entre 10 y 30 minutos dependiendo del tamaño de la tropa y de la visibilidad de los individuos.

Para evitar contar dos veces el mismo individuo en un fragmento, los integrantes de los GCM se separaron por al menos $50 \mathrm{~m}$ y se utilizó la aplicación OruxMaps para asegurarse de que todo el fragmento (especialmente los más grandes) fuera recorrido en forma de zigzag sin dejar espacios por explorar y/o recorrer la misma vereda dos veces. Al contar con mapas y la localización de las tropas en tiempo real se determinó la distancia entre ellas, de manera que tropas de tamaño y composición diferente fueran distinguidas a corta distancia. Para evitar contar dos veces el mismo individuo entre fragmentos o en días diferentes, los recorridos de un día determinado abarcaron los fragmentos más cercanos dejando los fragmentos más distantes para días subsecuentes.

\section{Descripción del hábitat}

Un fragmento de vegetación se definió como todo aquel parche que tiene una zona reducida del área total que puede tener diferente forma, tamaño y grado de aislamiento, ocupado por cualquier especie para la obtención de recursos (Arroyo-Rodríguez et al. 2007). En este estudio, un fragmento se delimitó como todo aquel parche arbóreo mayor o igual a 0.1 ha, ya que es el área mínima en la que se ha registrado la presencia del mono saraguato negro en Tabasco (Pozo-Montuy y Serio Silva 2007), sin embargo, el censo se realizó en toda el área arbolada para verificar la presencia o ausencia de saraguatos negros. Los fragmentos ocupados por la especie se clasificaron de acuerdo con las categorías de vegetación y sus equivalencias propuestas en la Tabla 1. Debido a la perturbación que presentan, los diferentes tipos de selva mediana se clasificaron como vegetación secundaria de selva mediana sin considerar su asociación con el pukté, corozo o palmares (Guerra-Martínez y Ochoa-Gaona, 2008). Para el manglar, se tomó en cuenta como el área arbórea disponible, pero no se consideró como un hábitat óptimo según lo observado en otras investigaciones (Rodrigues et al. 2019). La descripción y nombre común de las especies arbóreas presentes en los fragmentos fueron corroborados por consulta de expertos en botánica y de libros especializados en la flora de Tabasco (López-Mendoza 1980, Zamudio y Guadarrama 1985).

Toda la vegetación presente en la RBPC se digitalizó mediante el software Google Earth Pro con imágenes de satélite de alta resolución, cuya cobertura se clasificó y validó con observaciones en campo para la correcta determinación de los tipos de uso del suelo y vegetación. Posteriormente los datos obtenidos en formato $\mathrm{KMZ}$ se transformaron a archivos vectoriales por medio del software Arc Gis 10.6 y Qgis 2.14. A los fragmentos que se recorrieron durante los trabajos de campo, únicamente se les determinó el tipo de vegetación y la altura del dosel y posteriormente en gabinete se les determinó el área con ayuda de un Sistema de Información Geográfica (SIG). Al término del análisis se obtuvo una capa de fragmentos con sus características (tipo de vegetación y su cálculo el área), lo que representa el mapa actualizado del uso del suelo y vegetación de la RBPC.

\section{Análisis poblacional de Alouatta pigra}

El estado de conservación de la población de $A$. pigra en la RBPC se determinó mediante 11 parámetros poblacionales y de hábitat que se han empleado en estudios previos (Estrada et al. 2002a, Estrada et al. 2002b, van Belle y Estrada 2005, Bonilla-Sánchez et al. 2010, Mandujano-Rodríguez 2011). Los cuales fueron: El número de registros, que fue el total de puntos georreferenciados, registrados con ayuda de un GPS en coordenadas UTM, en los que fueron avistados los monos, independientemente de si se trató de una tropa o de individuos solitarios. La distribución actual se refiere a la proyección espacial de todos los registros obtenidos de saraguatos negros en un mapa de la RBPC a través de un Sistema de Información Geográfica (SIG). La abundancia corresponde al total de individuos de $A$. pigra observados, como individuos solitarios o como parte de 
Tabla 1. Tipos de vegetación reconocidos y las equivalencias utilizadas por otros autores. Todas las asociaciones de selva mediana se identificaron como vegetación secundaria de selva mediana debido a la perturbación que presentan actualmente.

\begin{tabular}{|c|c|c|c|c|}
\hline López Mendoza 1980 & Zamudio y Guadarrama 1985 & $\begin{array}{l}\text { Guerra-Martinez y } \\
\text { Gaona } 2006\end{array}$ & Ochoa & Estudio Actual \\
\hline $\begin{array}{l}\text { selva mediana subperennifolia } \\
\text { de pukté } \\
\text { selva mediana subperennifolia } \\
\text { de corozo, guano y huapaque } \\
\text { vegetación riparía }\end{array}$ & $\begin{array}{l}\text { selva mediana subperennifolia } \\
\text { de pukté }\end{array}$ & \multicolumn{2}{|c|}{$\begin{array}{l}\text { selva mediana subperennifolia } \\
\text { de pukté }\end{array}$} & $\begin{array}{l}\text { vegetación secundaría de } \\
\text { selva mediana }\end{array}$ \\
\hline $\begin{array}{l}\text { selva baja espinosa perennifo- } \\
\text { lia de tinto }\end{array}$ & Tíntales & \multicolumn{2}{|l|}{ selva baja de tinto } & selva baja espinosa de tinto \\
\hline manglar & Manglar & \multirow{2}{*}{\multicolumn{2}{|c|}{$\begin{array}{l}\text { manglar } \\
\text { comunidades de hidrófitas } \\
\text { pastizal } \\
\text { cuerpo de agua }\end{array}$}} & manglar \\
\hline comunidades de hidrófitas & vegetacion hidrófitas & & & $\begin{array}{l}\text { popal-tular } \\
\text { pastizal } \\
\text { cuerpo de agua } \\
\text { pastizal con árboles dispersos }\end{array}$ \\
\hline
\end{tabular}

una tropa. La densidad cruda resultó de dividir el número total de individuos registrados entre el área de la RBPC. La densidad poblacional se calculó dividiendo el número de individuos observados entre el área arbórea disponible en la RBPC. La densidad ecológica indica el resultado de dividir el número de individuos entre el área arbórea ocupada por la especie, es decir, se dividió la abundancia entre la sumatoria del área de los fragmentos en los que se corroboró la presencia de la especie.

La composición y estructura de la población se determinó mediante los siguientes parámetros. El tamaño promedio de la tropa correspondió al promedio y a la desviación estándar de todos los tamaños de tropa registrados, por lo que los registros de individuos solitarios se descartaron. El tamaño modal de la tropa se refirió al tamaño de tropa observado con mayor frecuencia entre la población. La composición social fue la forma de organización de las tropas de saraguatos de acuerdo con el número y sexo de los individuos adultos, para luego clasificar a la tropa como multimacho-multihembra, unimachomultihembra, multimacho-unihembra, unimachounihembra, parejas del mismo sexo y grupos de un solo sexo; las frecuencias con las que se observaron estas categorías de tropas se expresaron en porcentajes. La estructura poblacional se definió como la composición de la población total de monos saraguatos, de acuerdo con el sexo y edad de todos los individuos independientemente de si fueron observados estando solos o como parte de una tropa.
Las categorías de sexo y edad de los individuos se expresaron en porcentajes. Con la proporción de sexos se calculó el potencial reproductivo de las hembras, lo que también puede predecir la variación temporal del tamaño de la población. La proporción de sexos indicó la razón entre el número de machos adultos y el número de hembras adultas. Sin embargo, también se estimó entre machos y hembras que se clasificaron como juveniles e infantes (individuos sexualmente inmaduros). También se determinó la razón entre el número de hembras adultas y el número de individuos inmaduros.

\section{RESULTADOS}

\section{Tamaño, composición y estructura de la población de A. pigra}

La población total del mono saraguato negro en la RBPC estuvo formada por 1929 individuos, de los que 57 se registraron como individuos solitarios (4 hembras y 53 machos) mientras que el resto se agruparon en 362 tropas. El tamaño de las tropas varió de dos a 13 individuos, con tamaño promedio y desviación estándar ( $\pm \mathrm{SD}$ ) de $4.6 \pm 2.6$ individuos; con un tamaño moda de cuatro individuos. Para la composición de las tropas, se obtuvo que el $34.72 \%$ fueron tipo unimacho-multihembra, el $28.89 \%$ tipo unimacho-unihembra, el $26.11 \%$ tipo multimacho-multihembra y $7.22 \%$ tipo multimachounihembra.También se observaron parejas y tropas 
de un solo sexo, pero representaron solo el $2.22 \%$ y el $0.83 \%$ del total de tropas observadas. Tomando en cuenta el sexo y la edad de cada uno de los individuos registrados, la población total estuvo formada por 569 machos adultos (equivalente al $29.50 \%$ de la población total), 684 hembras adultas (35.46\%), 261 machos juveniles (13.53\%), 231 hembras juveniles (11.98\%), 81 machos infantes (4.20\%), 73 hembras infantes $(3.78 \%)$, y 30 infantes $(1.56 \%)$ a los que no se les pudo determinar el sexo. Al comparar el número de individuos de las diferentes categorías de sexo y edad, se determinó que por cada macho adulto hubo 1.2 hembras adultas. La proporción de hembras por macho en juveniles e infantes fue de 0.8 y 0.9 , respectivamente; mientras que la proporción de individuos inmaduros (infantes y juveniles) por cada hembra adulta fue de 1.0 .

\section{Descripción del hábitat y distribución de la población de saraguatos negros}

El $92.4 \%$ del área total de la RBPC corresponde a popal tular y ecosistemas acuáticos permanentes (lagunas, ríos y arroyos) mientras que sólo el $7.6 \%$ presenta vegetación arbórea (Tabla 2). Es decir, en la RBPC existen 22997.14 ha de vegetación arbórea distribuidas en 3646 fragmentos con tamaños que oscilan entre 0.001 ha de vegetación secundaria de selva mediana y 2448.03 ha de manglar; con tamaño promedio de los fragmentos de $6.31 \pm 61.26$ ha. Del total de fragmentos, en 218 se distribuyeron 352 tropas y 55 individuos solitarios. El área total de estos fragmentos ocupados por los saraguatos fue de 9054.81 ha mientras que su tamaño promedio fue de $41.54 \pm 205.28$ ha con intervalo de 0.001 a 2448.03 ha. En zonas de pastizal con árboles dispersos, cuya área en la RBPC es de 888.64 hectáreas, se registró la presencia de ocho tropas y dos individuos solitarios. También se observaron dos tropas de saraguatos en árboles aislados. Del área arbórea, el 59.7\% está representado por el manglar. Considerando la totalidad del área de la RBPC (302 706 ha) y excluyendo las áreas del manglar, debido a que no se considera como un hábitat idóneo para la especie, la vegetación arbórea disponible para los saraguatos es del 3.1\% con respecto al total de la RBPC. A pesar de la mínima área disponible, la población de este primate silvestre en la RBPC ocupó el 35\% (1 611.02 ha) del área disponible (9 $273 \mathrm{ha}$ ), la cual correspondió a vegetación secundaria de selvas medianas y selva baja espinosas de tinto (Figura 2).

Del total de monos saraguatos registrados, el $53.22 \%$ se observaron en áreas de selva baja espinosa de tinto, el $32.70 \%$ en vegetación secundaria de selva mediana, el $11.22 \%$ en manglar, el $2.39 \%$ en pastizal con árboles dispersos y el $0.48 \%$ de los individuos en árboles aislados. La altura promedio de los árboles en los que fueron observados los monos fue de $14.4 \pm 5.6 \mathrm{~m}$, mientras que las tropas e individuos se movieron a una altura promedio de $11.2 \pm 4.6 \mathrm{~m}$. Estos valores fueron similares entre los diferentes tipos de vegetación arbórea (Tabla 2). Durante los avistamientos, los saraguatos negros dieron diferentes usos a 36 especies y cuatro morfoespecies de flora. La especie más utilizada fue el tinto (Haematoxylum campechianum) con el $30.8 \%$ de los registros, seguida por el tucuy (Pithecellobium lanceolatum) y el cantemó (Albizia niopoides) (Tabla 3).

La densidad cruda, a nivel del área total de la RBPC (302 706 ha), fue de 0.006 individuos por ha. La densidad poblacional, con la totalidad de vegetación arbórea disponible en la RBPC (22 997.14 ha), fue de 0.08 individuos por ha. La densidad ecológica, considerando solo el hábitat ocupado ( 9 054.81), fue de 0.21 individuos por ha. La distribución de los saraguatos de acuerdo con el tamaño de los fragmentos indicó que el $10.42 \%$ de su población se registró en fragmentos menores a 1 ha, estos fragmentos pequeños representaron el $64.21 \%$ de los fragmentos en la RBPC pero los saraguatos usaron sólo el $2.35 \%$ de estos. El mayor porcentaje de la población $(32.76 \%)$ se distribuyó en fragmentos entre 1.1 a 10 ha, pero de 1094 fragmentos de estos intervalos de tamaño, los saraguatos ocuparon sólo el $9.95 \%$. Respecto a fragmentos más grandes entre 10.1 a 50 ha el $30.48 \%$ de la población de saraguatos estuvieron en el $25.49 \%$ de 153 fragmentos. Finalmente el $24.16 \%$ de la población de saraguatos tuvieron presencia en fragmentos mayores a 50 ha, de 
Tabla 2. Abundancia y densidad ecológica del mono saraguato negro (Alouatta pigra) en los diferentes tipos de vegetación.

\begin{tabular}{|c|c|c|c|c|c|c|c|}
\hline Tipo de vegetación & $\begin{array}{l}\text { Número de } \\
\text { fragmentos } \\
\text { ocupados }\end{array}$ & $\begin{array}{l}\text { Número de } \\
\text { fragmentos } \\
\text { desocupados }^{\star *}\end{array}$ & $\begin{array}{l}\text { Área } \\
\text { ocupada } \\
\text { (ha) }\end{array}$ & $\begin{array}{l}\text { Abundancia } \\
\text { (número de } \\
\text { individuos) }\end{array}$ & $\begin{array}{l}\text { Número } \\
\text { de } \\
\text { Tropas }\end{array}$ & Solitarios & $\begin{array}{c}\text { Densidad } \\
\text { Ecológica } \\
\text { (ind/ha) }\end{array}$ \\
\hline $\begin{array}{l}\text { Vegetación secundaria de selva me- } \\
\text { diana }\end{array}$ & 67 & 1057 & 817.733 & 692 & 118 & 19 & 0.83 \\
\hline Selva baja espinosa de tinto & 135 & 2093 & 793.282 & 984 & 191 & 32 & 1.14 \\
\hline Manglar & 16 & 278 & 7443.80 & 211 & 43 & 4 & 0.02 \\
\hline Pastizal con árboles dispersos ${ }^{*}$ & 9 & NA & 888.637 & 36 & 8 & 2 & 0.04 \\
\hline Árbol aislado & NA & NA & NA & 6 & 2 & 0 & NA \\
\hline Totales & 218 & & 9054.811 & 1929 & 362 & 57 & 0.21 \\
\hline
\end{tabular}

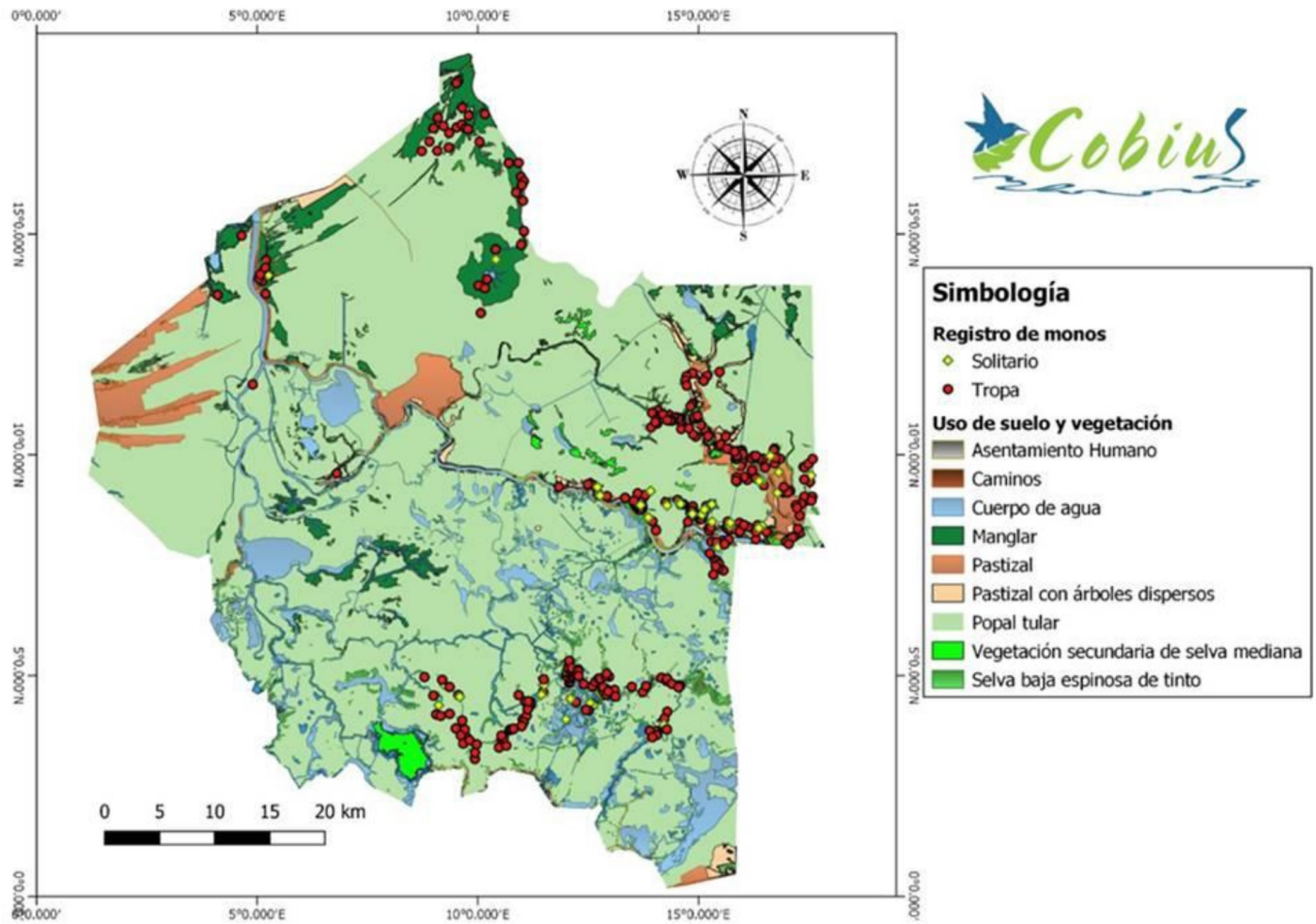

Figura 2. Distribución de la población de A. pigra en la Reserva de la Biosfera Pantanos de Centla y uso de tipo del suelo y vegetación del 2015 al 2018.

57 fragmentos con dichas dimensiones sólo usaron el $27.78 \%$.

\section{Abundancia, tasa de encuentro y densidad ecológica de $A$. pigra por localidad}

En el Ejido Marcos Díaz se registraron 680 individuos de A. pigra, en El Naranjo 295 individuos, y en el Ejido Tijeras 179 individuos (Tabla 4). El resto de los individuos $(\mathrm{N}=775)$ se distribuyeron entre ocho ejidos, localidades y rancherías que también forman parte de la RBPC. La longitud acumulada en kilómetros recorridos por localidad osciló entre $14 \mathrm{~km}$ en la localidad más pequeña y $249 \mathrm{~km}$ en la localidad más grande. La tasa de encuentro por localidad varió de 0.49 a 6.0 individuos por kilómetro recorrido. Considerando el hábitat ocupado, la densidad ecológica de saraguatos negros en el Ejido Tijeras fue de 1.51 individuos por ha, seguida de las densidades ecológicas estimadas para la Ranchería Chichicastle, El Naranjo y Tasajero, cuyos valores 
Tabla 3. Especies arbóreas más usadas por A. pigra en la Reserva de la Biosfera Pantanos de Centla y partes vegetales usadas como alimento (ítem).

\begin{tabular}{|c|c|c|c|c|}
\hline Especies & Nombre común & Frecuencia de uso & Consumida & Item consumido \\
\hline Haematoxylum campechianum & Tinto & 129 & $\mathrm{Si}$ & $\mathrm{HM}, \mathrm{HJ}, \mathrm{Fl}, \mathrm{Fr}$ \\
\hline Pithecellobium lanceolatum & Tucuy & 77 & $\mathrm{Si}$ & HJ, Ret, FI \\
\hline Albizia niopoides & Cantemó* & 27 & $\mathrm{Si}$ & $\mathrm{HJ}, \mathrm{Ret}, \mathrm{Fl}, \mathrm{Fr}$ \\
\hline Lonchocarpus spp. & Gusano & 25 & $\mathrm{Si}$ & $\mathrm{HJ}$, Ret, Fl, Fr \\
\hline Guazuma ulmifolia & Guazimo & 22 & $\mathrm{Si}$ & Ret, $\mathrm{Fr}$ \\
\hline Rhizophora mangle & Mangle rojo & 20 & No & - \\
\hline Tabebuia rosea & Macuilis & 17 & $\mathrm{Si}$ & HM, HJ, Ret, FI, Fr, Pec, Tallo \\
\hline Sabal mexicana & Guano & 15 & $\mathrm{Si}$ & $\mathrm{FI}, \mathrm{Fr}$ \\
\hline Casearia spp. & Botoncillo & 12 & No & - \\
\hline Laguncularia racemosa & Mangle negro & 10 & No & - \\
\hline Bucida burceras & Pukté & 9 & $\mathrm{Si}$ & Ret \\
\hline Spondias mombin & Jobo & 5 & $\mathrm{Si}$ & HM, HJ, Ret, Fr \\
\hline
\end{tabular}

Tabla 4. Abundancia, tasas de encuentro y densidad ecológica del mono saraguato negro por localidad monitoreada en la Reserva de la Biosfera Pantanos de Centla.

\begin{tabular}{llllll}
\hline Localidad & $\begin{array}{c}\text { Abundancia (número } \\
\text { de individuos) }\end{array}$ & $\begin{array}{c}\text { Longitud acumulada } \\
(\mathrm{km} \text { recorridos) }\end{array}$ & $\begin{array}{c}\text { Tasa de } \\
\text { Encuentro }\end{array}$ & $\begin{array}{c}\text { Área Ocupada } \\
\text { (ha) }\end{array}$ & $\begin{array}{c}\text { Densidad ecológica } \\
\text { (ind ha }\end{array}$ \\
\hline Ejido Marcos Díaz & 680 & 249.35 & 2.73 & 1682.47 & 0.40 \\
El Naranjo & 295 & 101.3 & 2.91 & 214.57 & 1.37 \\
Ejido Tijeras & 179 & 66.72 & 2.68 & 118.61 & 1.51 \\
Bitzal 7,5,3 & 170 & 62.92 & 2.70 & 152.72 & 1.11 \\
Tembladeras & 167 & 164.63 & 1.01 & 8798.71 & 0.02 \\
Ranchería Chichicastle & 113 & 19.3 & 5.85 & 80.01 & 1.41 \\
Ejido Mújica & 84 & 14 & 6.00 & 82.35 & 1.02 \\
Ribera Baja 2da Sección & 75 & 80.3 & 0.93 & 68.27 & 1.10 \\
Tasajero & 73 & 42.5 & 1.72 & 56.57 & 1.29 \\
El Palmar & 66 & 135.6 & 0.49 & 2123.41 & 0.03 \\
Elpidio Sánchez & 27 & 25 & 1.08 & 130.65 & 0.21 \\
Totales & 1929 & 961.62 & 2.56 & 13508.33 & 0.86 \\
\hline
\end{tabular}

fluctuaron entre 1.29 y 1.41 individuos por ha (Tabla 4).

\section{DISCUSIÓN}

\section{Tamaño, composición y estructura de la población de $A$. pigra}

Este fue el primer censo completo que se realizó sobre una población silvestre de $A$. pigra en un área Natural Protegida en Mesoamérica, que contó con la participación de grupos comunitarios de monitoreo formados por pobladores locales con amplio conocimiento de su territorio. Se logró determinar la presencia de 1929 individuos conformados en 362 tropas y 57 individuos solitarios, con tamaño promedio de tropas de $4.6 \pm 2.6$ individuos, proporción de 1.2 hembras por cada macho adultos y de un inmaduro por cada hembra adulta; cerca del $60 \%$ de las tropas estuvieron compuestas por un solo macho y varias hembras adultas. Estos valores de abundancia y estructura poblacional, tamaño y composición de las tropas, se encuentran dentro del intervalo de valores reportados para otros sitios fragmentados y continuos en Mesoamérica; en algunos casos, los valores encontrados han sido superiores a los datos de la Tabla 5.

En la región circundante al área de estudio se han realizado otros esfuerzos similares por censar a los saraguatos negros usando la misma metodología. Pero la población en la RBPC es la más grande reportada hasta la fecha. Uno de los primeros censos fue llevado a cabo por Pozo-Montuy et al. (2008), quienes registraron 1064 monos en casi 10000 ha de vegetación arbórea del municipio de Balancán, Tabasco. Para Playas de Catazajá, Chiapas, Bonilla- 
Tabla 5. Parámetros poblacionales del mono saraguato negro en sitios continuos y fragmentados en Mesoamérica 2002-2020.

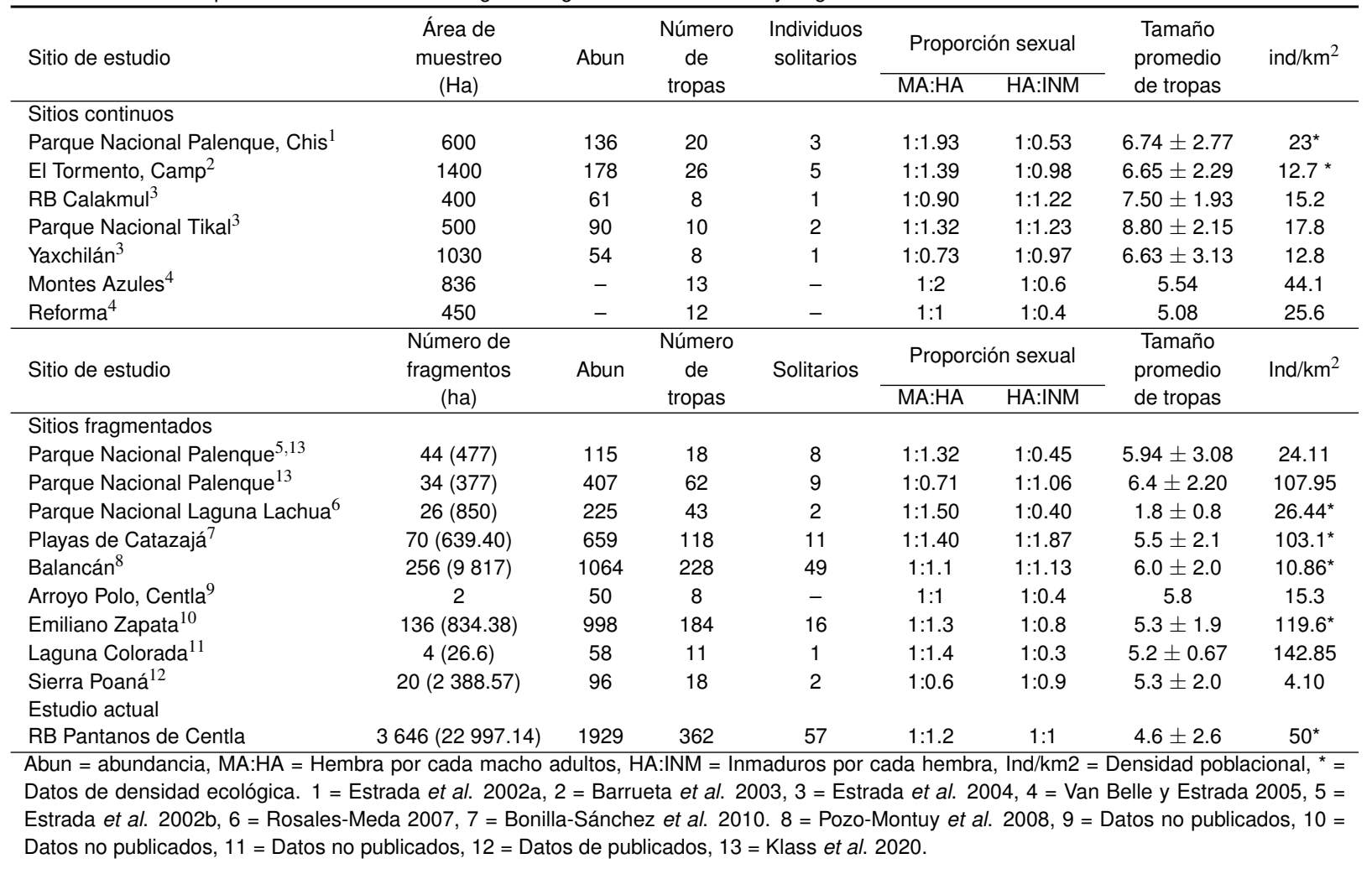

Sánchez et al. (2010) contabilizaron 659 monos en 70 fragmentos con un área total de 639.40 ha. En los últimos censos realizados en el estado de Tabasco se reportó una abundancia de 46 a 50 individuos de $A$. pigra en la comunidad de Arroyo Polo, en el municipio de Centla, y de 998 monos en poco más de 800 ha de vegetación forestal en el municipio de Emiliano Zapata. Con la integración de todos los censos realizados, se infiere que la cuenca baja del Usumacinta representa la región más importante por el tamaño de la población de $A$. pigra en toda su área de distribución en el sur-sureste de México, ya que la abundancia supera la de sitios más extensos como Calakmul y otras áreas protegidas de Campeche, Chiapas y Quintana Roo (Barrueta et al. 2003, Estrada et al. 2002a y 2002b, Estrada et al. 2004).

Los saraguatos negros son primates que, viven en tropas cohesivas, estables, y con patrones de organización que pueden ser entendidos con base a comportamientos de cooperación y competencia por el acceso y disputa de recursos, como el alimento o parejas sexuales (Sterck et al. 1997, Di Fiore y Campbell 2007). El valor del tamaño promedio de tropas (4.6 \pm 2.6 individuos) se encuentra dentro del intervalo, pero en el límite inferior reportado por otros autores en hábitat fragmentados. Al respecto, Horwich y Johnson (1986) mencionaron que el tamaño de tropas de $A$. pigra fue menos variable que en otros aulladores, puesto que típicamente la especie forma tropas menores a 12 individuos. En este sentido, otros autores han relacionado el tamaño de grupos a la densidad, considerando que en bajas densidades los grupos son pequeños y en densidades altas son grandes (Ostro et al. 2001). Sin embargo, Klass et al. (2020) reportaron que el tamaño de grupos es el resultado de una población que se reajusta en tropas cada vez más pequeñas en zonas de baja densidad, encontrando en un paisaje fragmentado altas densidades y grupos pequeños unimachos. Las estrategias de agrupamiento y de dispersión quizás estén más relacionadas en la RBPC con la tendencia de 
mantener grupos pequeños, pero con una alta dispersión de individuos como se evidencia con un alto número de individuos solitarios en comparación con otros sitios de estudio (Tabla 5).

En relación a la composición social, van Belle y Estrada (2005) consideraron que las tropas formadas por un macho y varias hembras fueron las más frecuentes de observar en poblaciones que habitan paisajes fragmentados y las tropas multimachomultihembra fueron frecuentes en poblaciones de selvas extensas. Mientras que Ostro et al. (2001) encontraron que los monos saraguatos negros también parecían preferir grupos más pequeños de un solo macho en condiciones de baja densidad de población, pero en densidades altas se encuentran comúnmente en grupos más grandes, con más machos por grupo, posiblemente porque no pueden establecer nuevos grupos en sitios reducidos por la fragmentación. Sin embargo, Klass et al. (2020) detectaron un patrón diferente en fragmentos con densidades altas y muy pocos grupos multimachos. En el caso de la RBPC, el $64 \%$ de las tropas son unimacho y el $33 \%$ multimacho por lo que puede estar influyendo la dispersión como mecanismo para evitar la competencia por recursos limitados y actuando a nivel de fragmento (Waser 1985).

Con base en el sistema socioecológico modificado por Sterck et al. (1997) los factores sociales y ecológicos como la agregación de las hembras determinada a su vez por el riesgo a la depredación, infanticidio, la distribución del alimento, la agregación de machos protectores elegidos por las hembras, pero sobre todo los regímenes competitivos influidos por la saturación del hábitat debido a la fragmentación y la ausencia de depredadores eliminados por los humanos pueden explicar la frecuencia de una composición unimacho (Gil Burmann 2002). Es decir, las hembras de saraguatos no tienen la necesidad de proteger sus recursos alimentarios por encontrarse dispersos, con baja probabilidad de infanticidio y la ausencia de depredadores hacen que la composición de un solo macho sea suficiente para su éxito reproductivo. Esto también tiene relación con la filopatría en las hembras, puesto que de los 57 registros de individuos solitarios 53 fueron machos adultos, lo cual sugiere que las hembras son las que se están quedando en los grupos y los machos son el sexo migrante.

La proporción relativa de individuos de cada sexo es uno de los parámetros más importantes para medir en una población puesto que afecta las estrategias reproductivas (Dias et al. 2020). En general, la proporción sexual concordó con los datos de composición social y tamaño de grupos, puesto que la población de saraguatos negros en la RBPC indicó una proporción mayor de una hembra por macho adulto (van Belle y Estrada 2008, Dias et al. 2015, Dias et al. 2016). Por su parte, el éxito reproductivo de los sistemas sociales presentes en la población de saraguatos se ve reflejado en la proporción 1:1 de inmaduros por cada hembra adulta. Lo que indica que, la composición social unimacho es suficiente para garantizar la continuidad reproductiva. A nivel mesoamericano, las proporciones sexuales en la RBPC se encuentran dentro de los intervalos conocidos para le especie tanto de sitios continuos y fragmentados (Tabla 5).

\section{Descripción del hábitat y distribución de la población de saraguatos negros}

Es importante señalar que, a pesar de la plasticidad conductual y ecológica de los saraguatos negros para habitar ambientes sumamente perturbados (Pozo-Montuy et al. 2013), los monos podrían no estar capacitados para mantener esa situación a largo plazo ya que, en las poblaciones en las que hay mayor competencia por los recursos disponibles deben asumir riesgos de depredación por perros y atropellamientos cuando descienden al suelo para aprovechar algunos recursos, como el agua, mientras se desplazan a otros fragmentos, además del riesgo de electrocutarse en las líneas de alta tensión cuando se mueven hacia las áreas urbanas para alimentarse en los huertos de los pobladores (van Belle y Estrada 2005, Bicca-Marques et al. 2019). En este sentido, se ha detectado una estrategia de distribución de los saraguatos en la RBPC, se observó que el $32.76 \%$ de la población habita en fragmentos de 1.1 a 10 ha, el $30.48 \%$ en fragmentos de 10 a 50 ha y el $24.16 \%$ en fragmentos mayores a 50 ha y solo el $10.42 \%$ de 
la población habita en fragmentos menores a 1 ha, a pesar de que el $64.21 \%$ de los fragmentos en la RBPC son de dicho tamaño. Al respecto, BiccaMarques et al. (2019), indican que las acciones de conservación de esta especie deben ser a escala regional considerando la flexibilidad de la especie para habitar o moverse en sitios fragmentados con una matriz enriquecida, como el aumento del número de árboles por hectárea, y tomando en cuenta a los fragmentos menores a una hectárea como trampolines, para garantizar la vida de la población a largo plazo.

\section{Abundancia, tasa de encuentro y densidad ecológica de $A$. pigra por localidad}

Ante los escenarios actuales de impactos ambientales y deterioro, es importante contar con una línea base o la generación de conocimiento para la toma de decisiones y planes de manejo para la protección de los recursos naturales en las ANPs (Ruiz-Montoya et al. 2017). Los estudios poblacionales y del hábitat de una especie o especies cobran relevancia cuando los manejadores necesitan tomar decisiones con base científica para dirigir los esfuerzos de conservación y el presupuesto de sus programas a zonas prioritarias de protección. Al respecto, es necesario no solo considerar el estado poblacional actual de una especie en toda el área de un ANPs, sino también determinar cuáles son las localidades, sitios y tipos de vegetación para la protección y conservación de la especie y su hábitat. Para el caso de la RBPC se determinó que los Ejidos Marcos Díaz, Tijeras, Bitzales, Tembladeras, Naranjo y Ranchería Chichicastle poseen los sitios más importantes para la conservación de los monos saraguatos y a las especies Haematoxylum campechianum, Pithecellobium lanceolatum y Albizia niopoides como las más usadas para alimentarse. Se observó que los estudios poblacionales son más eficientes al incorporar la participación de la población local tanto de primates como de otras especies, además es una ventaja tener aliados comunitarios para emprender actividades de restauración del hábitat y programas de desarrollo socioambiental a largo plazo.

\section{CONCLUSIONES}

La RBPC alberga cerca 2000 saraguatos negros, una de las poblaciones más abundantes de las que se tiene registro en México. Su estado poblacional es adecuado, debido a que presenta proporción de sexos y tamaño de tropas dentro de los intervalos específicos para la especie en sitios fragmentados y continuos. Además, de que tiene una densidad ecológica mayor que la de otros sitios conservados y entre las más altas en sitios fragmentados de Mesoamérica. La población habita en mayor porcentaje sobre la selva baja espinosa de tinto y en vegetación secundaria de selva mediana, con la excepción de, que las selvas medianas se encuentran en estado secundario debido a la perturbación. En la RBPC la selva baja espinosa de tinto y la vegetación secundaria de selva mediana representa oportunidades de crecimiento poblacional porque la ocupación actual es menor al 50\% del área arbórea. Por lo que es necesario emprender acciones para incrementar el área de los fragmentos menores a 1 ha debido a que son un poco más del $64 \%$ de los fragmentos de la reserva. Es necesario realizar investigaciones relacionadas con el seguimiento interanual de la población para detectar tendencias o fluctuaciones poblacionales, así como emprender monitoreos sobre las respuestas de comportamiento de estos primates ante la fragmentación y el nivel de conectividad del paisaje en la RBPC. Este trabajo muestra la importancia de contar con la colaboración de grupos comunitarios de monitoreo, debido a que su participación facilita la obtención de información, obtener permisos ejidales y privados, además de que conocen su territorio, lo que permite llegar hasta sitios de difícil acceso, que son ignorados cuando los monitoreos son realizados solo por investigadores.

\section{AGRADECIMIENTOS}

A la Comisión Nacional de Áreas Naturales Protegidas por el Subsidio PROCER 2015, 2016 y 2018 para llevar a cabo el presente trabajo. Al Ing. Carlos M. Villar Bedian exdirector de la RBPC, a la Dirección Regional Planicie Costera y Golfo de 
México a cargo del Biol. José Carlos Pizaña Soto y al Coordinador de subsidios PROCER Ing. Gabriela López Ramírez. También a los grupos de monitoreo del Ejido Marcos Diaz y Tijeras del Municipio de Jonuta, Tembladeras y El Palmar del Municipio de Centla, Rivera Baja y Bitzales Séptima de Macuspana, Tabasco; asi como a técnicos y voluntarios de COBIUS A.C.

\section{LITERATURA CITADA}

Arroyo-Rodríguez V, Mandujano S, Benitez Malvido J (2007) Landscape Attributes Affecting Patch Occupancy by Howler Monkeys (Alouatta palliata mexicana) at Los Tuxtlas, Mexico. American Journal of Primatology 70 : 69-77.

Barba Macias E, Valadez Cruz F, Pinkus M, Pinkus M, Juárez Flores J (2015) Reserva de la Biosfera Pantanos de Centla: Aspectos socioambientales prioritarios. En: Ortega-Rubio A, Pinkus-Rendón MJ, Espita-Moreno IC (ed) Las Áreas Naturales Protegidas y la Investigación Científica en México. Primera edición. Centro de Investigaciones Biológicas del Noroeste S.C. La Paz BCS. México. pp: 11-29.

Barrueta T, Estrada A, Pozo C, Calme S (2003) Reconocimiento demográfico de Alouatta pigra y Ateles geoffroyi en la Reserva El Tormento, Campeche, México. Neotropical Primates 11: 163-167.

Bicca-Marques JC, Chaves OM, Pacheco Hass G (2019) Howler monkey tolerance to habitat shrinking: Lifetime warranty or death sentence? American Journal of Primatology 82: 23089. Doi: 10.1002/ajp.23089.

Bonilla-Sánchez YM, Serio-Silva JC, Pozo-Montuy G, Bynum N (2010) Population status and identification of potential hábitats for the conservation of the endangered black howler monkey Alouatta pigra in northern Chiapas, Mexico. Oryx 44: 293-299.

Bonilla-Sánchez YM, Serio-Silva JC, Pozo-Montuy G, Chapman CA (2012) Howlers Are Able to Survive in Eucalyptus Plantations Where Remnant and Regenerating Vegetation Is Available. International Journal of Primatology 33: 233-245.

Busch J, Ferretti-Gallon K (2017) What drives deforestation and what stops it? A meta-analysis. Review of Environmental Economics and Policy 11(1): 3-23.

Calixto-Pérez E, Alarcón-Guerrero J, Ramos-Fernández G, Dias PAD, Rangel-Negrín A, Améndola-Pimenta M, Domingo C, Arroyo-Rodríguez v, Pozo-Montuy G, Pinacho-Guendulain G, Urquiza-Haas T, Koleff P, Martínez-Meyer E (2018) Integrating expert knowledge and ecological niche models to estimate Mexican primates' distribution. Primates 59: 451-467.

Carabias J, Provencio E, Maza J, Romero J (2000) Programa de manejo de la Reserva de la Biosfera Pantanos de Centla. Instituto Nacional de Ecología. México. 222p.

CONANP (2018) Memoria documental Entrega- recepción y rendición de cuentas 2012-2018 Dirección General de Conservación para el Desarrollo. www.conanp.gob.mx/InformeRendicion/ Memoriadocumental12.pdf. Fecha de consulta: 15 de Junio de 2020.

Cortés-Ortiz L, Bermingham E, Rico E, Rodríguez-Luna E, Sampaio L, Ruiz-García M (2003) Molecular systematics and biogeography of the Neotropical monkey genus, Alouatta. Molecular Phylogenetics and Evolution 26: 64-81.

Di Fiore A, Campbell CJ (2007) The aelines: variation in ecology, behavior, and social organization. In: Campbell J, Fuentes AF, MacKinnon KC, Panger M, Bearder S (ed) Primates in perspective. Volumen 1, Oxford University Press. United Kindom. pp: 155-185. 
Dias PAD, Coyohua-Fuentes A, Canales-Espinosa D, Rangel-Negrín A (2015) Group structure and dynamics in black howlers (Alouatta pigra): A 7-year perspective. International Journal of Primatology 36: 311-33.

Dias PAD, Rangel-Negrín A (2015) Diets of howler monkeys. In: Kowalewski M, Garber PA, Cortés-Ortiz L, Urbani B, Youlatos D (ed) Howler monkeys: Behavior, ecology, and conservation. Springer New York. USA. pp: 21-56.

Dias PAD, Coyohua-Fuentes A, Canales-Espinosa D, Rangel-Negrín A (2016) Factors influencing the reproductive success of female black howlers (Alouatta pigra). International Journal of Primatology 37: 638-655.

Dias PAD, Montero Domínguez IL, Rangel Negrín A (2020) Factors influencing infant sex ratio in howler monkeys (Alouatta spp.): A literature review and analysis. American Journal of Physical Anthropology 172: 48-57.

Dolder W, Dolder U (2009) Animales en peligro de extinción. Especies en vías de extinción y hábitats amenazados. Parragon. Barcelona, España. 256p.

Estrada A, Castellanos L, García Y, Franco B, Muñoz D, Ibarra A, Rivera A, Fuentes E, Jiménez C (2002a) Survey of tha black howler monkey (Alouatta pigra), population at the maya site of Palenque, Chiapas, México. Primates 44: 51-58.

Estrada A, Garber PA, Rylands AB, Roos C, Fernandez-Duque E, Di Fiore A, Nekaris KA-I, Nijman V, Heymann EW, Lambert JE, Rovero F, Barelli C, Setchell JM, Gillespie TR, Mittermeier RA, Arregoitia LV, de Guinea M, Gouveia S, Dobrovolski R, Shanee S, Shanee N, Boyle SA, Fuentes A, MacKinnon KC, Amato KR, Meyer ALS, Wich S, Sussman RW, Pan R, Kone I, Li B (2017) Impending extinction crisis of the world's primates: Why primates matter. Science Advances 3(1): e1600946. DOI: 10.1126/sciadv.1600946

Estrada A, Mendoza A, Castellano L, Pacheco R, Van-Belle S, García Y, Muñoz D (2002b) Population of the black howler monkey (Alouatta pigra) in a fragmented landscape in Palenque, Chiapas, México. American Journal of Primatology 58: 45-55.

Estrada A, Luecke L, Van Belle S, Barrueta E, Rosales-Meda M (2004) Survey of black howler (Alouatta pigra) and spider (Ateles geoffroyi) monkeys in the Mayan sites of Calakmul and Yaxchilan, Mexico and Tikal, Guatemala. Primates 45: 33-39.

Gil Burmann C (2002) Influencia ambiental en los sistemas sociales de primates. En: Martínez- Contreras J, Veá JJ (ed) Primates: Evolución, Cultura y Diversidad. Centro de Estudios Filosoficos, Politicos y Sociales "Vicente Lombardo Toledano". México. pp: 255-270.

Gouveia SF, Villalobos F, Dobrovolski R, Beltrão-Mendes R, Ferrari SF (2014) Forest structure drives global diversity of primates. Journal of Animal Ecology 83: 1523-1530.

Guadarrama-Olivera MA, Ortiz-Gil G (2000) Análisis de la flora de la Reserva de la Biosfera de los Pantanos de Centla, Tabasco, México. Universidad y Ciencia 15: 67-104.

Guerra-Martínez C, Ochoa-Gaona S (2006) Evaluación espacio-temporal de la vegetación y uso del suelo en la Reserva de la Biosfera Pantanos de Centla, Tabasco (1900-2000). Investigaciones geográficas, Boletín del Instituto de Geografía 56: 7-25.

Guerra-Martínez C, Ochoa-Gaona S (2008) Evaluación del programa de manejo de la reserva de la biosfera pantanos de Centla en Tabasco, México. Universidad y Ciencia 24: 135-146.

Horwich R, Johnson E (1986) Geographic distribution of the black howler monkey (Alouatta pigra) in Central America. Primates 27: 53-62.

Kitchen D (2004) Alpha male black howler monkey responses to loud calls: effect of numeric odds, male companion behaviour and reproductive investment. Animal behaviour 67: 125-139. 
Klass K, Van Belle S, Estrada A (2020) Demographic population structure of black howler monkeys in fragmented and continuous forest in Chiapas, Mexico: Implications for conservation. American Journal of Primatology 82: e23163. DOI: 10.1002/ajp.23163.

Lambert J (2010) Primate seed dispersers as umbrella species: A case study from Kibale National Park, Uganda, with implications for Afrotropical forest conservation. American Journal of Primatology 71: 1-16.

López- Jiménez LN, Jiménez- Lopez DA, Castillo-Acosta O, Gallardo- Cruz JA, Fernández- Montes de Oca AI (2020) Plantas vasculares de la Reserva de la Biosfera Pantanos de Centla, Mexico. Botanical Sciences 98: 159-204.

López- Mendoza R (1980) Tipos de vegetación y su distribución en el estado de Tabasco y Norte de Chiapas. Universidad Autónoma Chapingo. Centro Regional Tropical Puyacatengo. Dirección de Difusión Cultural. Mexico. 121p.

Macossay- Cortez A, Sánchez AJ, Florido R, Huidobro L, Montalvo-Urgel H (2011) Historical and environmental distribution of ichthyofauna in the Tropical wetland of pantanos de centla, southern Gulf of Mexico. Acta Ichthyologica et Piscatoria 41: 229-245.

Mandujano-Rodríguez S (2011) Ecología de poblaciones aplicada al manejo de fauna silvestre: cuatro conceptos $(\mathrm{N}, \lambda, \mathrm{MSY}, \mathrm{Pe})$. Instituto Literario de Veracruz. México. 104p.

Margules CR, Pressey RL (2000) Systematic conservation planning. Nature 405: 243.

Mouquet N, Gravel D, Massol F, Calcagno V (2013) Extending the concept of keystone species to communities and ecosystems. Ecology Letters 16: 1-8.

NRC (1981) Techniques for the Study of Primate Population Ecology.National Academy Press, Washington, D.C. $255 p$.

Ocampo HAG, Cortés-Calva P, Dávalos LII, Ortega-Rubio A (2014) Las áreas naturales protegidas de México. Investigación y Ciencia 22: 7-15.

Oropeza-Hernández P, Rendón-Hernández E (2012) Programa de acción para la conservación de las especies: primates, mono araña (Ateles geoffroyi) y monos saraguatos (Alouatta palliata, Alouatta pigra). Secretaría de Medio Ambiente y Recursos Naturales/Comisión Nacional de Áreas Naturales Protegidas. México. 53p.

Ortega-Álvarez R, Sánchez-González LA, Valera-Bermejo A, Berlanga-García H (2017) Community-Based Monitoring and Protected Areas: Towards an Inclusive Model. Sustainable Development 25: 200-212.

Ostro LET, Silver SC, Koontz FW, Horwich RH, Brockett R (2001) Shifts in social structure of black howler (Alouatta pigra) groups associated with natural and experimental variation in population density. International Journal of Primatology 22: 733-748.

Pavelka MSM, Knopff KH (2004) Diet and activity in black howler monkeys (Alouatta pigra) in southern Belize: does degree of frugivory influence activity level? Primates 45: 105-111.

Pozo-Montuy G, Serio-Silva JC (2007) Movement and resource use by a group of Alouatta pigra in a forest fragment in Balancán, México. Primates 48: 102-107.

Pozo-Montuy G, Serio-Silva JC, Bonilla-Sánchez M, Chapman CA (2013) Resource use in a landscape matrix by an arboreal primate: Evidence of supplementation in black howlers (Alouatta pigra). American Journal of Primatology 34: 647-860.

Pozo-Montuy G, Serio-Silva JC, Bonilla-Sánchez YM (2011) Influence of the landscape matrix on the abundance of arboreal primates in fragmented landscapes. Primates 52: 139-147. 
Pozo-Montuy G, Serio-Silva JC, Bonilla-Sánchez YM, Bynum N, Landgrave R (2008) Current Status of the Hábitat and Population of the Black Howler Monkey (Alouatta pigra) in Balancán, Tabasco, Mexico. American Journal of Primatology 70: 1169-1176.

Rodrigues dos Santos R, Bridgeman L, Supriatna J, Siregar R, Winarni N, Salmi R (2019) Behavioural ecology of mangrove primates and their neighbours. In: Nowak K, Barnett A, Matsuda I (Ed) Primates in flooded habitats: Ecology and conservation. Cambridge University Press. United Kingdom. pp: 124-133.

Romero GJ, García MA, Bautista JA, Pérez APH (2000) Caracterización de la Reserva de la Biosfera Pantanos de Centla. Universidad y Ciencia 15: 15-20.

Rondinini C, Wilson KA, Boitani L, Grantham H, Possingham HP (2006) Tradeoffs of different types of species occurrence data for use in systematic conservation planning. Ecology Letters 9: 1136-1145.

Rosales-Meda M, Estrada E, Lopez JE (2007) Demographic survey of black howler monkey (Alouatta pigra) in the Lachua Eco-Region in Alta Verapaz, Guatemala. American Journal of Primatology 69: 1-19.

Ruiz-Montoya L, Álvarez Gordillo G, Ramírez-Marcial N y Cruz-Salazar B (2017) Vulnerabilidad social y Biológica ante el Cambio Climático en la Reserva de la Biosfera Selva El Ocote. Colegio de la Frontera Sur. San Cristóbal de las Casas, Chiapas, México. 622p.

Sorensen TC, Fedigan LM (2000) Distribution of three monkey species along a gradient of regenerating tropical dry forest. Biological Conservation 92: 227-240.

Soria- Barreto M, González- Díaz AA, Castillo- Dominguez A, Álvarez Pliego N, Rodiles- Hernández R (2018) Diversidad ictica en la cuenca del Usumacinta, México. Revista Mexicana de Biodiversidad 89(suplemento): S100-S117.

Soulé ME (1985) What is conservation biology? BioScience 35: 727-734.

Sutherland WJ (2006) Ecological census techniques: a handbook: Cambridge university press. United Kingdom. $66 \mathrm{p}$.

Sterck EHM, Watts DP, van Schaik CP (1997) The evolution of female social relationships in non human primates. Behavioral Ecology and Sociobiology 41: 291-309.

Tobón W, Urquiza-Haas T, Ramos-Fernández G, Calixto-Pérez E, Alarcón-Guerrero J, Kolb M, Koleff P (2012) Prioridades para la conservación de los primates en México. Comisión Nacional para el Conocimiento y Uso de la Biodiversidad (CONABIO)-Asociación Mexicana de Primatología, A.C.-Comisión Nacional de Áreas Naturales Protegidas, Mexico. https://bioteca.biodiversidad.gob.mx/janium-bin/janium_zui.pl?jzd=/janium/ Documentos/ETAPA06/AP/7387/7387.jzd\&fn=7387. Fecha de consulta: 20 septiembre 2020.

Van Belle S, Estrada A (2005) Cambios demográficos en poblaciones del mono saraguato negro (Alouatta pigra) como consecuencia de la fragmentación del hábitat. Universidad y Ciencia. No. Esp. II: 1-9.

Van Belle S, Estrada A (2019) The influence of loud calls on intergroup spacing mechanism in black howler monkeys (Alouatta pigra). International Journal of Primatology 41: 265-286.

Van Belle S, Estrada A (2008) Group Size and Composition Influence Male and Female Reproductive Success in Black Howler Monkeys (Alouatta pigra). American Journal of Primatology 70: 1-7.

Waser PM (1985) Does competition drive dispersal? Ecology 66: 1170-1175.

Watts ES, Rico-Gray V (1987) Los primates de la Península de Yucatán, México: Estudio preliminar sobre su distribución actual y estado de conservación. Biótica 12: 57-66. 
Zamudio S, Guadarrama MA (1985) La vegetación actual de la cuenca del río Usumacinta en el estado de Tabasco. En: Gobierno del Estado de Tabasco (Ed) Usumacinta. Secretaría de Cultura y Recreación. Villahermosa, Tabasco. Mexico. pp: 9-75. 